\title{
Low molecular weight heparin in prevention of perioperative thrombosis
}

\author{
A Leizorovicz, M C Haugh, F-R Chapuis, M M Samama, J-P Boissel
}

\begin{abstract}
Objective-To determine whether prophylactic treatment with low molecular weight heparin reduces the incidence of thrombosis in patients who have had general or orthopaedic surgery.

Design-Meta-analysis of results from 52 randomised, controlled clinical studies (29 in general surgery and 23 in orthopaedic surgery) in which low molecular weight heparin was compared with placebo, dextran, or unfractionated heparin.

Subjects-Patients who had had general or orthopaedic surgery.

Intervention-Once daily injection of a low molecular weight heparin compared with placebo, dextran, or unfractionated heparin.

Main outcome measures-Incidence of deep venous thrombosis, pulmonary embolism, major haemorrhages, and death.

Results-The results confirm that low molecular weight heparins are more efficacious for the prophylactic treatment of deep venous thrombosis than placebo (common odds ratio $0.31,95 \%$ confidence interval 0.22 to $0.43 ; \mathrm{p}<0.001)$ and dextran $(0.44$, 0.30 to $0.65 ; p<0.001)$. The results suggest that low molecular weight heparins are also more efficacious than unfractionated heparin $(0.85,0.74$ to 0.97 $p=0.02$ ), with no significant difference in the incidence of major haemorrhages $(1.06,0.93$ to 1.20 ; $\mathrm{p}=\mathbf{0 . 6 2}$ ).

Conclusions-Low molecular weight heparins seem to have a higher benefit to risk ratio than unfractionated heparin in preventing perioperative thrombosis. However, it remains to be shown in a suitably powered clinical trial whether low molecular weight heparin reduces the risk of fatal pulmonary embolism compared with heparin.
\end{abstract}

\section{Introduction}

Patients undergoing surgery with prolonged general anaesthesia or a period of limited mobility postoperatively, or both, face the risk of thromboembolism. From phlebography and measurement of the uptake of fibrinogen labelled with iodine-125 the incidence of deep venous thrombosis in patients over 40 who have undergone general surgery is estimated to be between $20 \%$ and $30 \%$; this incidence is much higher in patients who have undergone orthopaedic surgery..$^{1-3}$ Although in many cases deep vein thrombosis resolves without sequelae once mobility is re-established, in some cases it can lead to valvar damage and chronic venous insufficiency and in rare cases to non-fatal or fatal pulmonary embolism from displacement of the thrombus.

Heparin, a naturally occurring oligosaccharide, has been used to treat thrombosis since the mid-1930s, and more recently it has been extensively evaluated in numerous clinical trials as a possible prophylactic treatment for deep vein thrombosis and pulmonary embolism in patients undergoing surgery. The incidence of pulmonary embolism is low, thus in many studies the incidence of deep vein thrombosis, which occurs more frequently, has been used as a surrogate end point.

Initially, major haemorrhagic complications were found to be a serious problem, but the use of a low dose regimen - that is, 5000 IU two or three times dailyhas reduced these. A recent overview of the results from more than 70 clinical trials with subcutaneously administered unfractionated heparin concluded that patients receiving treatment had a reduced incidence compared with control patients, of both deep vein thrombosis $(9.0 \% v 22.4 \%$; reduction of odds $67.0 \%$ $( \pm 4 \%) ; \mathrm{p}<0.001)$ and pulmonary embolism $(1.7 \% v$ $3.0 \%$; reduction of odds $47.0 \%(20 \%) ; \mathrm{p}<0.02){ }^{4}$

Dextran has also been used as a prophylactic treatment in this indication, and evidence suggest that it can reduce the incidence of pulmonary embolism in patients undergoing orthopaedic surgery. ${ }^{5}$ There is a low risk of anaphylactic reactions, but this is compensated by the haemodilution properties of dextran, which reduce the need for blood transfusion..$^{5}$

In recent years a better understanding of heparin's structure and mechanism of action has led to the development of new molecules of heparin with a lower molecular weight. These are obtained from native, purified heparin by one of four methods ${ }^{6}$ and they have a molecular weight varying from 3000 to 10000 daltons, depending on the manufacturer (unfractionated heparin is usually a mixture ranging from 5000 to 30000 daltons, with a mean of 12000 to 15000 daltons). These newer molecules have both biological and practical advantages-for example, they have an improved antithrombotic effect to bleeding ratio in animals, ${ }^{7-9}$ which is attributed to their ability to inhibit factor Xa, and affect the activated partial thromboplasmin time minimally. Low molecular weight heparins have a smaller disruptive effect on platelets compared with unfractionated heparin,,$^{10}$ and they are less effectively neutralised by platelet factor $4 .{ }^{11}$ From a practical point of view these molecules have an increased bioavailability compared with unfractionated heparin $(85 \% v 10 \%)$ and a half life that can vary from 3 to 18 hours - for example, in one study the half life for a particular low molecular weight heparin, fragmin, was found to be $3.7 \mathrm{~h}$, with activity remaining after $10 \mathrm{~h} .{ }^{12} 13$ In clinical medicine these differences enable the newer molecules to be administered once daily, unlike treatment with unfractionated heparin, which requires two or three injections a day.

These results gave rise to a hypothesis that the low molecular weight heparins could be more efficacious than unfractionated heparin for the prophylactic treatment of thrombosis, with a lower incidence of haemorrhagic complications and therefore a higher benefit to risk ratio. Many randomised, clinical trials have been undertaken to compare these molecules with placebo, 
dextran, and unfractionated heparin, and the apparently disparate results obtained may be explained by the low statistical power of the individual studies. Thus, the relative prophylactic efficacy of low molecular weight heparin compared with the other available treatments remains to be established.

We therefore reviewed all the available data from clinical trials comparing a low molecular weight heparin with placebo, unfractionated heparin, or dextran in patients undergoing general or orthopaedic surgery. Our aim was to examine the inherent and relative efficacy of these new heparin molecules in the prophylactic treatment of deep vein thrombosis in patients undergoing general and orthopaedic surgery.

\section{Methods}

DATA COLLECTION

We performed a literature search, both manual and computer aided (MEDLINE), for clinical trials evaluating a low molecular weight heparin in patients undergoing either elective or non-elective general or orthopaedic surgery from 1984 to 1991 , with no restriction on the language of the paper. We searched meeting abstracts, checked the International Society for Thrombosis and Haemostasis register, ${ }^{14}$ scanned the reference lists in reviews and studies, and asked colleagues, investigators, and the manufacturers of these products for any unpublished or missing studies. When studies were published both as an abstract and an original paper, only the paper was considered, and care was taken to eliminate duplicate reports.

Our inclusion criteria selected randomised, controlled studies which had used venous thrombosis of the lower limbs (detected by the fibrinogen uptake test, the thermographic DeVeTherm test, Doppler ultrasonography and phlebography) or pulmonary embolism, or both, as the clinical end point(s). We selected only trials with a control group, either untreated or treated with placebo, unfractionated low dose heparin, or dextran, and therefore dose ranging studies were excluded. Trials with at least one group treated with low molecular weight heparin were selected, but those evaluating the heparinoid OR10172 (Organon) were not because this preparation contains mainly unfractionated heparin sulphate and dermatan sulphate, with only a small proportion of low molecular weight heparin.

The data from the individual trials were extracted independently by two of us (AL and $\mathrm{MCH}$ ), using the following end points: venous thrombosis of the lower limbs, pulmonary embolism (both non-fatal and fatal), major bleeding, and total mortality. The definitions of venous thrombosis and pulmonary embolism, as specified in each report, were used for the individual studies. The definitions of major bleeding events used in the original papers were heterogeneous and we, therefore, decided to use the author's definition, when given, and to include bleeding requiring blood transfusion, reoperation, permanent discontinuation of treatment, or leading to death, or a combination of these four criteria, when no definition was given in the report. It would have been both impracticable and artificial to have attempted to obtain empirically a more standardised definition for major bleeding and so we chose a more pragmatic approach which we believe is closer to the clinical reality. The definitions of minor bleeding events were even more heterogeneous and thus more difficult to assess; because these events have fewer consequences in terms of therapeutic strategy they were not considered in the analysis.

\section{STATISTICAL METHODS}

The results from each trial were summarised on an intention to treat basis in two by two tables for each end point. A comparison of control groups between trials was performed using descriptive statistical methods and $\chi^{2}$ tests. The meta-analysis was performed using various techniques - that is, the combined logarithm of the odds ratio (both exact and approximate), Mantel-Haenszel, Cochran, Peto, percentage difference (both fixed and random effects models)..$^{15}$ The results obtained from the differen methods were similar and therefore only the resuls from the exact combined logarithm of the odds ratio method, with the corresponding $95 \%$ confiden $\overline{\bar{\alpha}}$ intervals, are presented. An odds ratio equal to indicates no difference between the treatments less than 1 indicates that low molecular weight heparm is better-that is, an odds ratio of 0.80 indicat $\Phi_{8}$ a $20 \%$ relative risk reduction-and greater than indicates that the control treatment is better. Associa tion and heterogeneity tests were performed fö each meta-analysis. ${ }^{17} \mathrm{~A}$ p value of 0.05 or less fro $\overrightarrow{\mathrm{m}}$ an association test is usually taken to be significa in the association test. In a meta-analysis the resules of the individual studies may be considered as bei heterogeneous when the $\mathrm{p}$ value from the heterogeneif test is less than or equal to $0 \cdot 05$. A non-significant value - that is, $p>0.05$-does not, however, indicage similarity - that is, homogeneity-but rather failuse to detect a difference.

Meta-analyses were performed using data from triats comparing low molecular weight heparin with placelo in general, orthopaedic, and all types of surgery. $\mathbb{A}$ comparison of low molecular weight heparin with dextran in orthopaedic surgery was also performed (f trials in general surgery were found). Other metganalyses were performed comparing low molecular weight heparin with unfractionated heparins with daga from general, orthopaedic, and both types of surgerpo. Another analysis was performed after removal of at data on the doses of low molecular weight heparin thi are currently considered to be too high (increased ri of side effects) or too low (decreased efficacy) in the studies involving comparison with unfractionated heparin in both types of surgery. This was do to evaluate the range of doses which are current recommended by each manufacturer, and therefore, the exact dose was dependent on the type of heparit. An additional analysis was performed using the data from results in which the first injection was given least 12 hours before or after the operation because has been suggested that this may reduce the incidenge of bleeding.

An exploratory analysis of the results in terms of yeQ̊ of publication was performed for all surgery comparing low molecular weight heparin and unfractionated heparin to see when the cumulative result from the studies became stable. This type of analysis can also show the influence of past protocols on new onesexample, the elimination of particularly high or low doses.

\section{Results}

We found nine studies in which low moleculeor weight heparin was assessed in comparison with placebo $^{18-26}$ and 39 with unfractionated heparin ${ }^{2} 0^{2}$ (M Samama, unpublished data); three of the studim with unfractionated heparin were reported by Samanta et al. ${ }^{27} \mathrm{We}$ also found four studies in which the efficacy was compared with that of dextran..$^{63-66}$ The 52 studies ( 29 for general surgery and 23 for orthopaedic surger ) found by our literature search are listed in table I with a summary of the basic characteristics of exci study. Two reports were written in French, ${ }^{2055}$ four $\stackrel{\mathrm{m}}{\mathrm{T}}$ German,,$^{343446}$ and the rest in English. Eight of these had appeared only as abstracts, ${ }^{1823242640535457}$ one was unpublished, and the rest were original reports. Two 


\begin{tabular}{|c|c|c|c|c|c|c|c|c|c|c|c|c|c|}
\hline \multirow[b]{3}{*}{$\begin{array}{l}\text { Comparisun for low molecular } \\
\text { weight heparin treatment }\end{array}$} & \multirow[b]{3}{*}{ Name of heparin } & \multirow[b]{3}{*}{ Type of surgery } & \multicolumn{2}{|c|}{$\begin{array}{c}\text { Daily regimen } \\
\text { dose: : first iniecturont; duration of treatment }\end{array}$} & \multirow[b]{3}{*}{ End point assessment } & \multicolumn{8}{|c|}{ End pont (no of events no of pattents randomised in group: } \\
\hline & & & & & & \multicolumn{2}{|c|}{ Deep vein thrombowis } & \multicolumn{2}{|c|}{ Blecding } & \multicolumn{2}{|c|}{ Pulmunary embolism } & \multicolumn{2}{|c|}{ Total murrality } \\
\hline & & & 1.MWH group & Cintrol group & & $\begin{array}{l}\text { LMWH } \\
\text { group }\end{array}$ & $\begin{array}{c}\text { Control } \\
\text { grour }\end{array}$ & $\underset{\text { group }}{\text { LMwiH }}$ & $\underset{\substack{\text { Control } \\
\text { group }}}{-}$ & $\underset{\text { group }}{\operatorname{LMWH}}$ & $\underset{\substack{\text { Control } \\
\text { group }}}{-}$ & 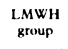 & $\underset{\substack{\text { Control } \\
\text { group }}}{-}$ \\
\hline \multicolumn{14}{|c|}{ (ienerul surgery } \\
\hline $\begin{array}{l}\text { Placebo: } \\
\text { Le Gagneux 1987\%" }\end{array}$ & Enoxaparin & Prostatectomy & & Placelero & FUT phleterography & $0 / 44$ & 0.45 & 8144 & $6 / 45$ & & & ND & \\
\hline $\begin{array}{l}\text { Ockelfirerd 1989." } \\
\text { Pezzuol, 1989" }\end{array}$ & $\begin{array}{l}\text { Fragmin } \\
\text { Fraxinarice }\end{array}$ & General & $1 \times 2500 ; 1-2 \mathrm{~h}$ beforere $5-9 \mathrm{day}$, & $\begin{array}{l}\text { Placebor } \\
\text { Pacento }\end{array}$ & FUT & $+1 / 102$ & 14.95 & $\begin{aligned} 4 / 102 \\
173247\end{aligned}$ & $\begin{array}{l}4 / 95 \\
692251\end{array}$ & 0 & 2995 & $0: 0102$ & $\underset{18 / 2251}{2125}$ \\
\hline $\begin{array}{l}\text { Gezhle 1988 } \\
\text { Unfractionated hep }\end{array}$ & $\begin{array}{l}\text { Frappartinc } \\
\text { Fluxum }\end{array}$ & 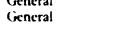 & $\begin{array}{l}17500: 2 \mathrm{~h} \mathrm{hetore;} ; 7.21 \text { days } \\
1 \times 7500: 2 \mathrm{~h} \text { before: } 7 \text { days }\end{array}$ & $\begin{array}{l}\text { Placetoo } \\
\text { Placetoo }\end{array}$ & 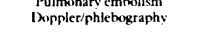 & 0,50 & 3:50 & $\begin{array}{c}173 / 2247 \\
6.50\end{array}$ & 60 & 0,50 & $\begin{array}{l}8,2251 \\
0,501\end{array}$ & 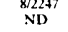 & \\
\hline $\begin{array}{l}\text { Unfractionated he } \\
\text { Samama } 1988\end{array}$ & Enoxaparin & Gineral & 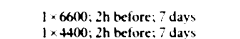 & $3 \times 5000: 2 \mathrm{~h}$ before: 7 days & FuT & 271600 & $19: 147$ & 71,160 & $6+1 / 47$ & $0 / 160$ & $1 / 4+7$ & $2 / 160$ & $0,0 / 177$ \\
\hline Samama 1988 & & & 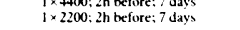 & 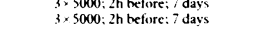 & $\begin{array}{l}\text { FUT } \\
\text { FUT }\end{array}$ & $\begin{array}{lll}241 / 168 \\
158\end{array}$ & $\begin{array}{l}156123 \\
21 / 167\end{array}$ & 71/168 & $\begin{array}{l}48 / 23 \\
58 / 167\end{array}$ & 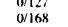 & $\begin{array}{l}0,123 \\
0 / 167\end{array}$ & 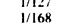 & $\begin{array}{l}0.123 \\
0 / 167\end{array}$ \\
\hline Verardi $1988^{\circ}$ & Fluxum & General & 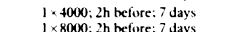 & 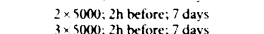 & FUT/Doppler/SGP & $10 / 300$ & $19 / 302$ & 3,308 & 111302 & 1/308 & $3 / 302$ & ND & \\
\hline 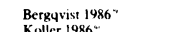 & Fragmin & Abduminal & $1 \times 5000$ 2h heforere $5-7$ days & 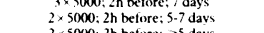 & FUT & $13 / 215$ & 9:217 & $55 / 215$ & $75 / 217$ & $0 / 215$ & $1 / 217$ & $5 / 215$ & $5 / 217$ \\
\hline $\begin{array}{l}\text { Koller 1 1986" } \\
\text { Onarheim 1986" }\end{array}$ & 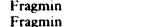 & $\begin{array}{l}\text { Visceral } \\
\text { Addominal }\end{array}$ & 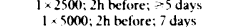 & 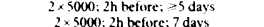 & $\begin{array}{l}\text { FTT/phlechography } \\
\text { FUT/2htlebogranhy }\end{array}$ & $2 / 75$ & $1 / 75$ & 5,775 & 2775 & 00775 & $1 / 75$ & 年, 0175 & $\begin{array}{l}0,75 \\
0.27\end{array}$ \\
\hline $\begin{array}{l}\text { Bergyvist } 1988^{\prime \prime} \\
\text { Borstad 1988" }\end{array}$ & $\begin{array}{l}\text { Fragmin } \\
\text { Fragmin }\end{array}$ & $\begin{array}{l}\text { Andominal } \\
\text { Gynaecological }\end{array}$ & $\begin{array}{l}1 \times 5000 ; 12 \text { b before, }>6 \text { days } \\
1 \times 5000 ; \text { in before: }>\text { days }\end{array}$ & 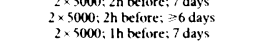 & 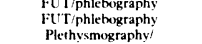 & $\begin{array}{l}28,505 \\
0.105\end{array}$ & $\begin{array}{l}2 / 27 \\
41 / 497 \\
0110\end{array}$ & $\begin{array}{l}2,255 \\
30,505 \\
601 / 105\end{array}$ & $\begin{array}{l}1272 \\
15 / 497 \\
55 / 110\end{array}$ & $\begin{array}{l}0,595 \\
0,505 \\
0,105\end{array}$ & $\begin{array}{l}0 / 27 \\
1 / 497 \\
0 / 110\end{array}$ & $\begin{array}{ll}0,305 \\
10 / 505\end{array}$ & $\begin{array}{l}10 / 497 \\
10 / 49\end{array}$ \\
\hline $\begin{array}{l}\text { Briel 1988" } \\
\text { (Gen }\end{array}$ & Fragmin & Ginaceculogical & $2 \times 2500$ day 1.1 .5000 days $2-8$ & $2 \times 5000+0.5 \mathrm{mg}$ DHE: 8 days & 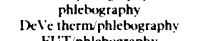 & ${ }_{1099}^{1994}$ & & & $2 / 101$ & & & & \\
\hline $\begin{array}{l}\text { Caen } 1988^{\prime} \\
\text { Fricker 1988 }\end{array}$ & $\begin{array}{l}\text { Fragmin } \\
\text { Fragmin }\end{array}$ & $\begin{array}{l}\text { Ciencral } \\
\text { Oncolugical }\end{array}$ & $\begin{array}{l}1,2500 ; \text {, th before: } 7 \text { days } \\
2 \times 2500 \text { day } 1,1 \times 5000 \text { davs } 2-10\end{array}$ & $\begin{array}{l}2 \times 5000: 2 \mathrm{~h} \mathrm{before:} 7 \text { days } \\
3 \times 5000 ; 2 \mathrm{~h} \text { before; } 10 \text { days }\end{array}$ & $\begin{array}{l}\text { FUT'phletwography } \\
\text { FLT'phlebography }\end{array}$ & $\begin{array}{l}6,145 \\
0,40\end{array}$ & $\begin{array}{l}7190 \\
0 ; 40\end{array}$ & $\begin{array}{l}11 / 195 \\
2 / 40\end{array}$ & $\begin{array}{l}8 / 190 \\
1 / 40\end{array}$ & $\begin{array}{r}0 / 195 \\
0: 40\end{array}$ & $\begin{array}{l}1 / 190 \\
2 / 40\end{array}$ & $\begin{array}{l}2: 195 \\
0,40\end{array}$ & $\begin{array}{l}3 / 190 \\
0,40\end{array}$ \\
\hline $\begin{array}{l}\text { Hart 1990, } \\
\text { Kakkar 1985" }\end{array}$ & $\begin{array}{l}\text { Fragmin } \\
\text { Fraxiparine }\end{array}$ & $\begin{array}{l}\text { Ahduminal } \\
\text { Athduminal }\end{array}$ & $\begin{array}{l}1 \times 2500 ; 2 \mathrm{~h} \text { hefore } \\
1 \times 7500 ;: 2 \mathrm{~h} \text { hefore } ; \text { days } \\
1,75 \text { days }\end{array}$ & $\begin{array}{l}2 \times 5000 ; 2 \mathrm{~h} \mathrm{before} ; 7 \text { days } \\
2 \times 5000: 2 \mathrm{~h} \text { before: } \geqslant 7 \text { days }\end{array}$ & $\begin{array}{l}\text { FUT/phlebography } \\
\text { FUT/phletoggraphy }\end{array}$ & $\begin{array}{l}6 / 26 \\
5 / 2120\end{array}$ & $\begin{array}{r}6124 \\
151200\end{array}$ & $\begin{array}{r}1 / 126 \\
102000\end{array}$ & $\begin{array}{l}3 / 124 \\
7 / 200\end{array}$ & $\begin{array}{l}1 / 126 \\
0 / 200\end{array}$ & $\begin{array}{l}1 / 124 \\
1 / 200\end{array}$ & $\begin{array}{l}5 / 126 \\
5 / 200\end{array}$ & $\begin{array}{l}3 / 124 \\
6 / 200\end{array}$ \\
\hline $\begin{array}{l}\text { EFS Group 1988" } \\
\text { Dahan 1989"* }\end{array}$ & $\begin{array}{l}\text { Fraxparine } \\
\text { Fraxiparine }\end{array}$ & $\begin{array}{l}\text { Abdominal } \\
\text { Oncological }\end{array}$ & $\begin{array}{c}1 \times 7500 ; 2 \text { heffore; } 7 \text { days } \\
7500 \text { day } 1-3,10000 \text { days } 4.7\end{array}$ & $\begin{array}{l}3 \times 5000: \text { : 2h befure, } 7 \text { days } \\
3 \times 5000 \text { day } 1-3, \text { ND days } 3-7 ; 2 \mathrm{~h} \text { before }\end{array}$ & $\begin{array}{l}\text { FUT'phlchoorraphy } \\
\text { FUT/phletography }\end{array}$ & $\underset{0,50}{27 / 968}$ & $\begin{array}{l}42,941 \\
0,50\end{array}$ & $\begin{aligned} 150 / 968 \\
3 / 50\end{aligned}$ & $\begin{array}{c}1+1 / 941 \\
2 / 50\end{array}$ & $\begin{array}{l}27968 \\
0 / 50\end{array}$ & $\begin{array}{l}5 / 941 \\
0,50\end{array}$ & $\stackrel{11 / 968}{\mathrm{ND}}$ & $12 / 4+1$ \\
\hline $\begin{array}{l}\text { Samama (unpublished) } \\
\text { Leizorovicz [991" }\end{array}$ & $\begin{array}{l}\text { Logiparin } \\
\text { Logiparin }\end{array}$ & $\begin{array}{l}\text { General } \\
\text { Gieneral }\end{array}$ & $\begin{array}{l}12 \mathrm{~h} \mathrm{before} \\
1 \times 2500 ; 2 \mathrm{~h} \mathrm{before:} 7-10 \text { days } \\
1 \times 2500 ; 2 \mathrm{~h} \text { before and } 12 \mathrm{~h} \text { after; }\end{array}$ & $\begin{array}{l}2 \times 50000 ; 2 \mathrm{~h} \mathrm{befiore;} ; 7-10 \text { days } \\
2 \times 5000 ; 2 \mathrm{~h} \text { beture to } 12 \mathrm{~h} \text { after } ; 7-10 \text { days }\end{array}$ & $\begin{array}{l}\text { FUT } \\
\text { FUT/phlekerraphy }\end{array}$ & $\begin{array}{l}0: 18 \\
23: 861\end{array}$ & $\begin{array}{l}0,19 \\
7 / 429\end{array}$ & $\begin{array}{l}0: 18 \\
22886\end{array}$ & $\begin{array}{c}1 / 19 \\
14429\end{array}$ & $\underset{5 / 81}{N 181}$ & $2 / 429$ & $\begin{array}{l}1 / 1 / 18 \\
201861\end{array}$ & $\begin{array}{l}0,194 \\
9 / 429 \\
9\end{array}-30$ \\
\hline Schmizz-Huebrer $1984^{\circ}$ & Sandoz & Abduminal & 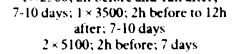 & & (1) & $3: 84$ & 042 & $117 / 84$ & $0 / 42$ & $0 / 84$ & $0 / 42$ & $1 / 84$ & $0 / 42$ \\
\hline Adulf 1989. & Sandor & Aldominnal & $\begin{array}{l}2 \times 6000 ; \text { th before } 7 \text { days } \\
1 \times 6300\end{array}$ & $3 \times 5000 ; 2 \mathrm{~h} \mathrm{befure:} \geqslant 7$ davs & & 3,587202 & 24,202 & 63202 & $4 \times 202$ & 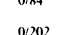 & $8,4 i^{12}$ & & \\
\hline $\begin{array}{l}\text { dddell 1989" } \\
\text { H Heilmann 1989." }\end{array}$ & $\begin{array}{l}\text { Sandoz } \\
\text { Sandoz }\end{array}$ & $\begin{array}{l}\text { Ahdominal } \\
\text { Gynaccologital }\end{array}$ & 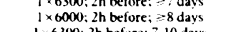 & 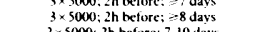 & 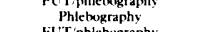 & 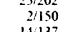 & 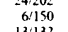 & $\begin{array}{ll}6521 / 150 \\
3 / 10\end{array}$ & 2,150 & ND & $2 / 202$ & O:150 & 0,150 \\
\hline $\begin{array}{l}\text { Sasahara 1986 } \\
\text { Vivigt } 1986^{\circ}\end{array}$ & $\begin{array}{l}\text { Sandoz }+0.5 \mathrm{mg} \mathrm{DHE} \\
\text { Sandoz }+0.5 \mathrm{mg} \mathrm{DHE}\end{array}$ & $\begin{array}{l}\text { Adblominal } \\
\text { Aldstomnal }\end{array}$ & $\begin{array}{l}1 \times 6300 ; 2 \mathrm{~h} \text { before; } ;-10 \text { days } \\
\times 630 ;\end{array}$ & $\begin{array}{l}2 \times 5000 ; 2 \mathrm{~h} \mathrm{before;} ; 7.10 \text { days } \\
2 \times 5000\end{array}$ & $\begin{array}{l}\text { FUT phleboug } \\
\text { Phichurar }\end{array}$ & $\begin{array}{c}1+13737 \\
1,103\end{array}$ & $\frac{13 / 132}{1 / 199}$ & $19: 137$ & $\begin{array}{l}31 / 132 \\
197\end{array}$ & 00137 & $\frac{2 / 132}{1 / 97}$ & $\begin{array}{l}2: 137 \\
4 / 103\end{array}$ & 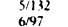 \\
\hline $\begin{array}{l}\text { Toigr } 1986^{*} 1999^{\circ} \\
\text { Baumgarterer }\end{array}$ & $\begin{array}{l}\text { Sandoz }+0.5 \mathrm{mg} \text { DHF: } \\
\text { Sandoz }+0.5 \mathrm{mg} \mathrm{DHE}\end{array}$ & $\begin{array}{l}\text { Aldominal } \\
\text { Atduminal }\end{array}$ & & $\begin{array}{l}2 \times 3000 ; 2 \mathrm{~h} \text { betorec: } \\
2 \times 250 ;\end{array}$ & $\begin{array}{l}\text { Phlcowgraphy } \\
\text { F(T//phlebography }\end{array}$ & 6,999 & 3102 & 0,999 & $0 / 102$ & $1 / 99$ & $1 / 102$ & 年, 199 & $2 / 102$ \\
\hline Kakkar $19899^{\circ}$ & Sandoz + $05 \mathrm{mg}$ DHI: & Atdominal & 1. $\times 6300 ;$ ih befure $\geqslant 7$ days & $\begin{array}{l}2 \times 5000 ; 2 \mathrm{~h} \text { before, } \geqslant 7 \text { days } \\
\text { Orthopuedic surgery }\end{array}$ & FUT/fhlebography & $8 / 88$ & 10991 & $0: 88$ & 0,91 & 2888 & 0/91 & ND & \\
\hline $\begin{array}{l}\text { Placebvo: } \\
\text { Turpie 1986: }\end{array}$ & Enoxaparin & Elective hip & $2 \times 2400 ; 12-24$ a after & 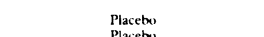 & v & 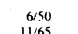 & $21 / 50$ & $1 / 50$ & $2: 50$ & $0 / 50$ & $0 / 50$ & 0,50 & $1 / 50$ \\
\hline $\begin{array}{l}\text { Leclerc } 1991 . \\
\text { Jorgensen } 1989 .\end{array}$ & $\begin{array}{l}\text { Enoxaparin } \\
\text { Fragnin }\end{array}$ & $\begin{array}{l}\text { Knec } \\
\text { Hip fracture }\end{array}$ & $\begin{array}{l}2 \times 30 \mathrm{mg}: 12-24 \mathrm{~h} \text { after; } ; \mathrm{ND} \\
2 \times 2500 \text { : } 2 \mathrm{~h} \text { before to } 12 \mathrm{~h} \text { after: }\end{array}$ & $\begin{array}{l}\text { Placetory } \\
\text { Placetoro }\end{array}$ & $\begin{array}{l}\text { FlT/phlebography } \\
\text { FL'T/phlebography }\end{array}$ & $\begin{array}{r}11 / 65 \\
9 / 30\end{array}$ & $\begin{array}{l}37 / 64 \\
22 / 38\end{array}$ & $\begin{array}{l}465 \\
\mathrm{NS}\end{array}$ & & $\underset{0 / 30}{N D}$ & 1/38 & $\begin{array}{c}\mathrm{ND} \\
0 / 30\end{array}$ & $1 / 38$ \\
\hline Torholm 1991 & Fragmin & Eiletive hip & $\begin{array}{l}1 \times 5000: 6 \text { days } \\
2 \times 2500: 2 \mathrm{~h} \mathrm{hefore} \mathrm{to} 12 \mathrm{~h} \text { after: }\end{array}$ & Placetor & FET/phletwography & 9:58 & $19: 54$ & NS & & 0,58 & $1 / 54$ & 1/58 & $0 / 54$ \\
\hline Lassen 1989* & Lugiparin & Electure hip & $\begin{array}{l}1 \times 5000 ; 6 \text { days } \\
1 \times 50 \mathrm{Cl} / \mathrm{kg}: 2 \mathrm{~h} \text { before: } \mathrm{ND}\end{array}$ & Placetor, & Phletugraphy & 2790 & $45: 102$ & $0 / 90$ & $1 / 102$ & NI) & & 190 & 1/102 \\
\hline $\begin{array}{l}\text { Dextran: } \\
\text { DES Group 1991." }\end{array}$ & Enoxaparin & Electure hup & 1. 3900: $12 \mathrm{~h} \mathrm{before:} 7$ days & $60 \mathrm{mg} / \mathrm{ml}: 500 \mathrm{ml}:$ : wice on day 0. & Phictougraphy & 7120 & $2+126$ & is & & $0 / 120$ & 0,126 & $1 / 120$ & $0 / 126$ \\
\hline Erikswon 1988" & Fragmin & Electrve hip & $2 \times 2500: 2 \mathrm{~h} \mathrm{befure} ; 7$ day & $\begin{aligned} \text { once on days } 1 \\
\text { Dextran } 702 \times 500\end{aligned}$ & Fl T/phlectography & 1050 & $22: 50$ & osso & $0: 50$ & $2 / 50$ & $2 / 50$ & $0 / 50$ & 0,50 \\
\hline Matzsch 1988 & Logiparin & Electrve hip & 1. $35 \mathrm{~kg}:$ : h beliure: 7 days & Dextran $70500 \mathrm{ml}$ before and after & FLT/phlebugraphy & $13 / 48$ & 1952 & $0 / 48$ & 0/52 & 0,48 & 0/52 & $0 / 48$ & 0,52 \\
\hline Matzwh 1991" & Lugiparin & Elective hip & 1. $50 \mathrm{~kg}:$ : h hefore: 7 days & $\begin{array}{l}\text { surgervy and on dayy } 1,3, \text { and } 5 \\
\text { Dextran } 70500 \text { mi before and after } \\
\text { surgery and on days } 1,3, \text { and } 5\end{array}$ & H.T/phletography & 22120 & $36 / 123$ & +120 & 10/123 & $6: 120$ & $0 / 123$ & $0: 120$ & 0,123 \\
\hline $\begin{array}{l}\text { Unfractionated heparin: } \\
\text { Planes 1988" } \\
\text { Levine 1991" }\end{array}$ & $\begin{array}{l}\text { Enoxaparin } \\
\text { Enoxaparin }\end{array}$ & $\begin{array}{l}\text { Elective hip } \\
\text { Eilective hip }\end{array}$ & 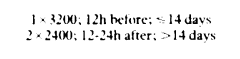 & $\begin{array}{l}3 \times 5000: 2 \mathrm{~h} \text { beture } \leq 14 \text { days } \\
2 \times 7500 \text { (1: } 12 \text { or } 24 \text { h beture, }>1+\text { days }\end{array}$ & 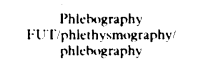 & $\begin{array}{l}15: 124 \\
57 / 333\end{array}$ & $\underset{27 / 113}{63132}$ & $\begin{array}{r}2 / 124 \\
11 / 333\end{array}$ & $\begin{array}{r}01113 \\
191332\end{array}$ & $\begin{array}{l}0 / 124 \\
0 / 333\end{array}$ & $\begin{array}{l}1 / 113 \\
2 ; 332\end{array}$ & $\begin{array}{l}0,124 \\
0,133\end{array}$ & $\begin{array}{l}0: 1133 \\
0,1332\end{array}$ \\
\hline $\begin{array}{l}\text { Chiapuzeno } 1988 \\
\text { Pin 1 } 1989\end{array}$ & $\begin{array}{l}\text { Fluxum } \\
\text { Fluxum }\end{array}$ & $\begin{array}{l}\text { Elective hip } \\
\text { Hip }\end{array}$ & 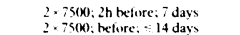 & 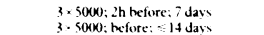 & 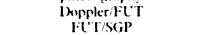 & $\begin{array}{l}5 / 70 \\
5 / 25\end{array}$ & $7 / 20$ & $\begin{array}{l}0770 \\
0 / 25\end{array}$ & $\begin{array}{l}0 / 70 \\
0 / 24\end{array}$ & $\begin{array}{l}0,70 \\
0.25\end{array}$ & $\begin{array}{l}0,70 \\
1 / 24\end{array}$ & $\begin{array}{c}\mathrm{ND} \\
0.25\end{array}$ & $1: 24$ \\
\hline Haas 1985 & Fragmin & lilective hip & $2 \cdot 2500$ dar 0.1 .5000 dave $1-15$ & $2 \times 5000+$ DHF: 2 h hetore: 15 daw & FIT & 665 & $7 / 65$ & is & & 165 & $0 / 65$ & Ni) & \\
\hline $\begin{array}{l}\text { Binsack } 1986^{\circ} \\
\text { Barre 1987 }\end{array}$ & $\begin{array}{l}\text { Fragmin } \\
\text { Fragmin }\end{array}$ & $\begin{array}{l}\text { Elective hip } \\
\text { b.jective hip }\end{array}$ & 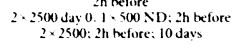 & $\begin{array}{l}\text { 3. } 50000 \text { : 2h heforee : ND } \\
\text { 3. } 50000 \mathrm{C}: \text { day } 0 . \text {. ND days 1-10: }\end{array}$ & $\begin{array}{l}\text { FUT phletengraphy } \\
\text { Phlebergraphy }\end{array}$ & $\begin{array}{l}5,48 \\
7+40\end{array}$ & $\begin{array}{l}6+7 \\
+1+0\end{array}$ & $\begin{array}{l}0+48 \\
6+40\end{array}$ & $\begin{array}{l}0,47 \\
+40\end{array}$ & $\begin{array}{l}\mathrm{ND} \\
0.40\end{array}$ & $0 / 40$ & 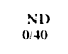 & $0 / 40$ \\
\hline Dechavanne $1989^{\circ}$ & Fragmin & Elective hip & $\begin{array}{l}2 \times 2500 \text { for } 10-13 \text { days or } 2 \times 2500 \\
0.50-0,3)\end{array}$ & $2 \times 5000 ;$ days 1.2, NDD days $3-13$ & F: $:$ /phletergraphy & $5: 82$ & $+4+0$ & $0: 82$ & $0 / 40$ & N1) & & 0,82 & 0:40 \\
\hline $\begin{array}{l}\text { Erikssun } 1989 \\
\text { Monreal } 1989 .\end{array}$ & $\begin{array}{l}\text { Fragmin } \\
\text { Fragmin }\end{array}$ & $\begin{array}{l}\text { Elective hip } \\
\text { Hip }\end{array}$ & 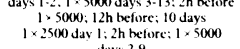 & 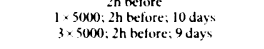 & $\begin{array}{l}\text { Phlebugraphy } \\
\text { Phlethography }\end{array}$ & $\begin{array}{l}19667 \\
14+46\end{array}$ & $\begin{aligned} 25 / 69 \\
6 / 4+4\end{aligned}$ & $\begin{array}{l}1 / 67 \\
2 / 46\end{array}$ & $\begin{array}{l}5 / 69 \\
1 / 44\end{array}$ & $\begin{array}{l}8: 67 \\
6 / 46\end{array}$ & $\begin{array}{r}19 / 69 \\
0 / 44\end{array}$ & $\begin{array}{l}0,67 \\
2 / 46\end{array}$ & $\begin{array}{l}1 / 69 \\
3 / 44\end{array}$ \\
\hline Leyvraz 1991. & Fraxiparine & Elective hip & $1 \times 11 / \mathrm{kg}$ davi $1.3 .3 .1 \times 62 / \mathrm{kg}$ dav $+10 ;$ & N1); 2 th befure: 10 days & Phickugraphy & $22 / 203$ & $28 / 2066$ & $1 / 203$ & 3/2066 & 1/203 & +206 & 1/203 & $2 / 206$ \\
\hline 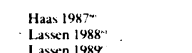 & $\begin{array}{l}\text { Sandur }+0.5 \mathrm{mg} \text { DHE } \\
\text { Sandor }+0.5 \mathrm{mg} \mathrm{DHE} \\
\text { S DHeto }\end{array}$ & 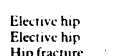 & 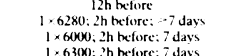 & 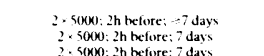 & 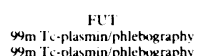 & $\begin{array}{l}15,80 \\
35 / 118 \\
1468\end{array}$ & $\begin{array}{l}15,1 / 10 \\
34122 \\
2,131\end{array}$ & $\begin{array}{l}26.80 \\
968118 \\
\text { Sts }\end{array}$ & $\begin{array}{l}13: 80 \\
981122\end{array}$ & $\begin{array}{l}0.00 \\
0,118 \\
0,118 \\
1 / 18\end{array}$ & $\begin{array}{l}1: 80 \\
0,122 \\
0,122\end{array}$ & $\begin{array}{l}0,80 \\
0: 118 \\
0,118\end{array}$ & $\begin{array}{l}0,000 \\
0,122 \\
0,121\end{array}$ \\
\hline & & & & & & & & & & & & & \\
\hline
\end{tabular}

studies in which dihydroergotamine was given to only one group were not included ${ }^{67}{ }^{68}$ as this resulted in noncomparable groups.

A summary of the results for the four end points is also given in table I. Although some letters were sent to investigators, not all information was retrievable or available - that is, not given in the reports or indicated as not significant - which is denoted in table I. Data on a total of 18543 patients were collected; $14567 \mathrm{had}$ undergone general surgery (29 studies) and 3976 orthopaedic surgery (23 studies). Studies comparing low molecular weight heparin with placebo involved 5479 patients ( 4884 (four studies) and 595 (five studies) undergoing general and orthopaedic surgery, respectively) and those comparing low molecular weight and unfractionated heparins involved 12375 patients ( 9683 ( 25 studies) and 2692 (14 studies) undergoing general and orthopaedic surgery, respectively). Only 689 patients were included in four studies comparing dextran with low molecular weight heparin in patients undergoing orthopaedic surgery. The total, unadjusted results for the various end points for the different type of surgery and type of control treatment used are summarised in table II.

\section{VERSUS PLACEBO}

Only one of the four studies in general surgery showed a significant reduction in deep vein thrombosis (a fifth study did not use deep vein thrombosis as an end point). All five studies in orthopaedic surgery showed a significant reduction between the treated and control groups.

The results obtained from the meta-analyses for the four end points are presented in table III. The incidence of deep vein thrombosis was significantly reduced for both types of surgery, with a common odds ratio of $0.25(95 \%$ confidence interval 0.09 to 0.70 $\mathrm{p}=0.008)$ for general surgery and $0.32(0.22$ to 0.46 ; $\mathrm{p}<0.001$ ) for orthopaedic surgery (fig 1 ). The overall odds ratio from the combined data was $0.31(0.22$ to $0.43 ; \mathrm{p}<0.001$ ) (figs 1 and 2 ). The $\mathrm{p}$ value for homogeneity was found to be high for each analysis $(p>0 \cdot 1)$, indicating that the treatment effect was not different, although the low molecular weight heparins used in the various studies had been produced by different companies, and the study populations were different..$^{70}$

The odds ratio for the incidence of pulmonary embolism in patients having general surgery was 0.33 $(0.09$ to 1.12$)$ and in those having orthopaedic surgery $0.64(0.08$ to 5.03$)$ (table III), but these results were not significant ( $p=0.07$ and $p=0.67$, respectively). The analysis for the combined results gave an odds ratio of $0.39(0.13$ to 1.12$)$, which was not significant $(p=0.08)$ because of the very large $95 \%$ confidence interval (table III). Although this is a clinically important result, the total number of patients included in these analyses, in other words the statistical power, was not sufficient to show a significant decrease, even if it existed.

The incidence of bleeding was significantly higher in 


\begin{tabular}{|c|c|c|c|c|c|c|c|c|}
\hline \multirow[b]{3}{*}{ Control treatment } & \multicolumn{8}{|c|}{ End point } \\
\hline & \multicolumn{2}{|c|}{ Deep vein thrombosis } & \multicolumn{2}{|c|}{ Pulmonary embolism } & \multicolumn{2}{|c|}{ Major bleeding } & \multicolumn{2}{|c|}{ Total mortality } \\
\hline & LMWH group & Control group & LMWH group & Control group & LMWH group & Control group & LMWH group & Control group \\
\hline \multicolumn{9}{|c|}{ General surgery } \\
\hline $\begin{array}{l}\text { Placebo } \\
\text { Unfractionated heparin }\end{array}$ & $\begin{array}{r}4 / 196(2 \cdot 04) \\
248 / 5108(4 \cdot 86)\end{array}$ & $\begin{array}{r}17 / 190(8 \cdot 95) \\
267 / 4575(5 \cdot 84)\end{array}$ & $\begin{array}{r}2 / 2443(0 \cdot 09) \\
12 / 4841(0 \cdot 25)\end{array}$ & $\begin{array}{l}10 / 2441(0 \cdot 41) \\
25 / 4305(0.58)\end{array}$ & $\begin{array}{l}191 / 2443(7 \cdot 82) \\
649 / 5108(12 \cdot 71)\end{array}$ & $\begin{array}{c}85 / 2441(3 \cdot 48) \\
596 / 4575(13 \cdot 03)\end{array}$ & $\begin{array}{r}8 / 2349(0 \cdot 34) \\
71 / 4253(1 \cdot 67)\end{array}$ & $\begin{array}{l}20 / 2346(0 \cdot 85) \\
60 / 3719(1 \cdot 61)\end{array}$ \\
\hline \multicolumn{9}{|c|}{ Orthopaedic surgery } \\
\hline $\begin{array}{l}\text { Placebo } \\
\text { Unfractionated heparin } \\
\text { Dextran }\end{array}$ & $\begin{array}{r}62 / 293(21 \cdot 16) \\
224 / 1369(17 \cdot 82) \\
52 / 338(15 \cdot 38)\end{array}$ & $\begin{array}{r}144 / 308(46 \cdot 75) \\
256 / 1323(19 \cdot 35) \\
101 / 351(28 \cdot 77)\end{array}$ & $\begin{array}{l}0 / 138 \\
17 / 1239(1 \cdot 37) \\
8 / 338(2 \cdot 37)\end{array}$ & $\begin{array}{r}2 / 142(1 \cdot 41) \\
28 / 1236(2 \cdot 27) \\
2 / 351(0 \cdot 57)\end{array}$ & $\begin{array}{c}5 / 205(2 \cdot 44) \\
145 / 1236(11 \cdot 73) \\
4 / 218(1 \cdot 83)\end{array}$ & $\begin{array}{l}8 / 217(3 \cdot 69) \\
2 / 351(12 \cdot 05) \\
10 / 225(4 \cdot 44)\end{array}$ & $\begin{array}{l}2 / 228(0 \cdot 88) \\
7 / 1186(0 \cdot 59) \\
0 / 338\end{array}$ & $\begin{array}{l}3 / 244(1 \cdot 23) \\
9 / 1141(0 \cdot 79) \\
0 / 351\end{array}$ \\
\hline \multicolumn{9}{|c|}{$\begin{array}{l}{ }^{\star} \text { Number of patients with outcome/total number of patients randomised. } \\
\text { LMWH = Low molecular weight heparin. }\end{array}$} \\
\hline & \multicolumn{8}{|c|}{ End point } \\
\hline & \multicolumn{2}{|c|}{ Deep vein thrombosis } & \multicolumn{2}{|c|}{ Pulmonary embolism } & \multicolumn{2}{|c|}{ Bleeding } & \multicolumn{2}{|c|}{ Total mortality } \\
\hline Type of surgery & $\begin{array}{l}\text { No of studies/No } \\
\text { of patients }\end{array}$ & $\begin{array}{l}\text { Odds ratio }(95 \% \\
\text { confidence } \\
\text { interval) }\end{array}$ & $\begin{array}{l}\text { No of studies/No } \\
\text { of patients }\end{array}$ & $\begin{array}{c}\text { Odds ratio }(95 \% \\
\text { confidence } \\
\text { interval) }\end{array}$ & $\begin{array}{l}\text { No of studies/No } \\
\text { of patients }\end{array}$ & $\begin{array}{l}\text { Odds ratio }(95 \% \\
\text { confidence } \\
\text { interval })\end{array}$ & $\begin{array}{l}\text { No of studies/No } \\
\text { of patients }\end{array}$ & $\begin{array}{l}\text { Odds ratio }(95 \% \\
\text { confidence } \\
\text { interval })\end{array}$ \\
\hline \multicolumn{9}{|c|}{ Placebo } \\
\hline General & $3 / 386$ & $0.25(0.09$ to 0.70$)$ & $4 / 4884$ & $0.33(0.09$ to 1.13$)$ & $4 / 4884$ & $2.35(1.80$ to 3.06$)$ & $2 / 4695$ & $0.42(0.19$ to 0.95$)$ \\
\hline Orthopaedic & $5 / 601$ & $0.32(0.22$ to 0.46$)$ & $3 / 280$ & $0.64(0.08$ to 5.03$)$ & $\begin{array}{l}4 / 4004 \\
3 / 422\end{array}$ & $0.69(0.22$ to $2 \cdot 11)$ & $4 / 472$ & $0.92(0.18$ to 4.62$)$ \\
\hline Both & $8 / 987$ & $0.31(0.22$ to 0.43$)$ & $7 / 5164$ & $0.39(0.13$ to $1 \cdot 12)$ & $7 / 5306$ & $2 \cdot 20(1.70$ to 2.85$)$ & $6 / 5167$ & $0.50(0.24$ to 1.02$)$ \\
\hline \multicolumn{9}{|c|}{ Unfractionated heparin } \\
\hline General & $25 / 9683$ & $0.86(0.72$ to 1.04$)$ & $22 / 9146$ & $0.62(0.33$ to 1.15$)$ & $25 / 9683$ & $1.02(0.90$ to 1.16$)$ & $19 / 7972$ & $0.96(0.68$ to 1.36$)$ \\
\hline Orthopaedic & $14 / 2692$ & $0.83(0.68$ to 1.02$)$ & $12 / 2475$ & $0.53(0.27$ to 1.03$)$ & $12 / 2423$ & $1.09(0.76$ to 1.58$)$ & $11 / 2327$ & $0.88(0.37$ to 2.07$)$ \\
\hline Both & $39 / 12375$ & $0.85(0.74$ to 0.97$)$ & $34 / 11621$ & $0.59(0.37$ to 0.93$)$ & $37 / 12106$ & $1.06(0.93$ to 1.20$)$ & $30 / 10299$ & $0.95(0.69$ to 1.31$)$ \\
\hline \multicolumn{9}{|c|}{ Dextran } \\
\hline Orthopaedic & $4 / 689$ & $0.44(0.30$ to 0.65$)$ & $3 / 443$ & $1.88(0.46$ to $7 \cdot 74)$ & $3 / 443$ & $0.45(0.15$ to 1.35$)$ & & \\
\hline
\end{tabular}

TABLE IV-Occurrence of major bleeding in studies with early or late first injection. Values are numbers of patients with outcome/total number of patients randomised

\begin{tabular}{|c|c|c|c|}
\hline Reference & $\begin{array}{c}\text { Time of injection (before } \\
\text { or after surgery) }\end{array}$ & $\begin{array}{l}\text { Low molecular weight } \\
\text { heparin group }\end{array}$ & Control group \\
\hline \multicolumn{4}{|c|}{ General surgery } \\
\hline v Placebo: & & & \\
\hline Le Gagneux ${ }^{1 \mathrm{x}}$ & $12 \mathrm{~h}$ before & $8 / 44$ & $6 / 45$ \\
\hline$v$ Unfractionated heparin: & $12 \mathrm{~h}$ before & $30 / 505$ & $15 / 497$ \\
\hline \multicolumn{4}{|c|}{ Orthopaedic surgery } \\
\hline$v$ Unfractionated heparin: & & & \\
\hline Planes $^{49}$ & $12 \mathrm{~h}$ before & $2 / 120$ & $0 / 108$ \\
\hline Eriksson" & $12 \mathrm{~h}$ before & $1 / 67$ & $5 / 69$ \\
\hline Leyvraz & $12 \mathrm{~h}$ before & $1 / 203$ & $3 / 206$ \\
\hline \multicolumn{4}{|l|}{ v Placebo: } \\
\hline Turpie" & $12 \mathrm{~h}$ after & $1 / 50$ & $2 / 50$ \\
\hline Leclerc: & $12 \mathrm{~h}$ after & $4 / 65$ & $5 / 65$ \\
\hline \multicolumn{4}{|l|}{$v$ Unfractionated heparin: } \\
\hline Levine $^{6 / 10}$ & $12 \mathrm{~h}$ after & $11 / 333$ & $19 / 332$ \\
\hline
\end{tabular}

the treated group compared with the control group in patients having general surgery (odds radio $2.35(1.80$ to 3.06$) ; \mathrm{p}<0.001$ ), whereas in patients having orthopaedic surgery the difference, although indicating an excess in the low molecular weight heparin group, was not significant (odds ratio $0.69(0 \cdot 22$ to $2 \cdot 11) ; p=0.52$ ). The analysis of the incidence of total mortality in both types of surgery gave an odds ratio of $0.50(0 \cdot 24$ to $1.02 ; p=0.053)$, which is on the borderline of significance.

\section{VERSUS DEXTRAN}

Only four studies comparing low molecular weight heparin with dextran in 689 patients undergoing orthopaedic surgery were found. Two of these studies showed a significant reduction in the incidence of deep vein thrombosis in the low molecular weight heparin group, but no difference was reported for the other end points. Meta-analysis showed a highly significant reduction for deep vein thrombosis in the patients receiving low molecular weight heparin, with an odds ratio of $0.44(0.30$ to $0.65 ; \mathrm{p}<0.001)$, and a nonsignificant trend for a reduction in bleeding (odds ratio
$0.45(0.15$ to 1.35$) ; p=0 \cdot 15)$. The incidence of $\vec{\bullet}$ pulmonary embolism was higher in the low molecular? weight heparin group (odds ratio $1 \cdot 88$ ), but the $95 \%$ confidence interval was large $(0.46$ to $7 \cdot 74 ; p=0.61)$, the difference seeming to be due to one trial. ${ }^{66}$

\section{VERSUS UNFRACTIONATED HEPARIN}

Four of the 25 general surgery studies ${ }^{28383941}$ and $\stackrel{2}{\rightleftharpoons}$ three of the 14 orthopaedic surgery studies ${ }^{49} 5758$ showed $\frac{}{3}$ a significant reduction $(p<0.05)$ in the incidence of deep vein thrombosis, as reported in the publications. The meta-analysis using the results from the trials for both types of surgery combined showed a significant reduction in the incidence of deep vein thrombosis in favour of the low molecular weight heparin group, with 3 an odds ratio of $0.85(0.74$ to $0.97 ; \mathrm{p}=0.02)$. A nonsignificant trend towards a reduction in the risk of deep vein thrombosis in patients treated with low molecular weight heparin was observed in patients undergoing 5 general surgery (odds ratio $0.86(0.72$ to 1.04$)$; N $\mathrm{p}=0 \cdot 12)$ and in those undergoing orthopaedic surgery (odds radio $0.83(0.68$ to 1.01$) ; \mathrm{p}=0.07)$ (table III).

We also considered the results from trials involving $N$ orthopaedic surgery in which phlebography was N systematically used for the confirmation of deep vein 0 thrombosis as the fibrinogen uptake test is generally considered to be unsuitable in these patients. After the removal of four trials from the initial analysis, ${ }^{50-5259}$ we $\stackrel{\odot}{+}$ found the odds ratio unchanged at 0.83 , with a marginally different $95 \%$ confidence interval $(0.68$ to $1 \cdot 02)$ and $p$ value $(p=0.09)$.

When the data for general and orthopaedic surgery $\frac{\Omega}{\mathbb{D}}$ patients were combined, the odds ratio for pulmonary embolism was 0.59 in favour of the low molecular weight group $(0.37$ to $0.93 ; p=0.02)$. The reduction in the incidence of pulmonary embolism in patients having orthopaedic surgery (odds ratio $0.53(0.27$ too $1.02) ; p=0.06$ ) was not significant because of the low incidence of the event coupled with the insufficient number of patients. For patients undergoing general surgery the result was similar, and although there were 
almost four times as many patients in the analysis, this number was still not sufficient (odds ratio 0.62 (0.33 to $1 \cdot 15) ;(p=0 \cdot 12)$. No significant differences were found between the two groups for bleeding complications $(\mathrm{p}>0.6)$ and total mortality $(\mathrm{p}>0.55)$ (table III).

In an analysis performed using data corresponding to the manufacturer's current recommended doses of the various low molecular weight heparins and for both types of surgery we found an odds ratio of $0.83(0.72$ to $0.95 ; p=0.007)$ for deep vein thrombosis and an odds ratio of $1.05(0.93$ to 1.20$)$ for major bleeding complications. The results for general surgery were similar, with the result for deep vein thrombosis being significant (odds ratio $0.80(0.66$ to 0.97$) ; p=0.02$ ) whereas that for major bleeding complications was unchanged (odds ratio $1.05(0.91$ to 1.21$)$ ) in comparison with the result for all doses (table III).

Five studies, two in general surgery ${ }^{1831}$ and three in orthopaedic surgery ${ }^{49} 57^{59}$ had a first injection 12 hours before surgery and three others ${ }^{22} 23{ }^{50}$ had a first injection 12 hours after orthopaedic surgery (table IV). We performed a meta-analysis using the data on the incidence of major bleeding from three orthopaedic surgery trials in which the first injection was given 12 hours before surgery ${ }^{49559}$ and compared the result with that from a meta-analysis using similar data from trials in which this injection was given two hours before surgery. ${ }^{5152545557586061}$ The first analysis showed a nonsignificant trend $(p=0.24)$ in favour of low molecular weight heparin (odds ratio 0.44 ), but the $95 \%$ confidence interval was large $(0 \cdot 11$ to $1 \cdot 77)$. The second analysis gave an odds ratio of $1.50(0.96$ to 2.32$)$, which is slightly more in favour of unfractionated heparin, compared with the result for all data (odds ratio 1.09

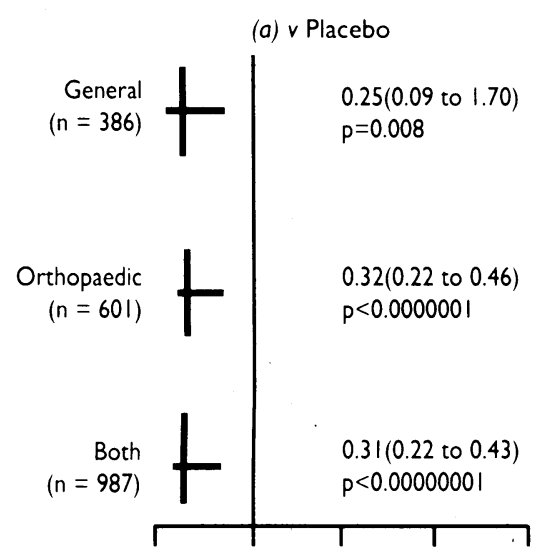

(c) $v$ Placebo

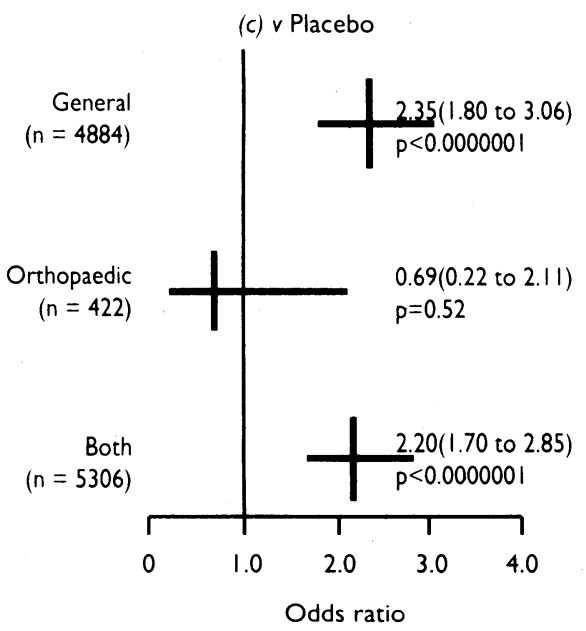

FIG 1-Graphical representation of results from meta-analyses (exact odds ratio method) for deep venous thrombosis $((a)$ and $(b))$ and major bleeding in $((c)$ and $(d))$ in general surgery, orthopaedic surgery, and both types of surgery. Odds ratio of $<1$ indicates that low molecular weight heparin is better than unfractionated heparin and of $>1$ that unfractionated heparin is better than low molecular weight heparin. Horizontal lines represent $95 \%$ confidence intervals; if value of 1 is included results are not significant

(b) $v$ Unfractionated heparin

$p=0.12$

$0.83(0.68$ to 1.02$)$ $p=0.07$

$0.85(0.74$ to 0.97$)$ $p=0.02$

(d) $\vee$ Unfractionated heparin
Schmitz-Huebner ${ }^{42}$

$\mathrm{Kakkar}^{38}$

Haas $^{53}$

Sasahara $^{45}$

Koller $^{30}$

Bergqvist $^{29}$

Onarheim $^{31}$

Samama (unpublished)

Binsack $^{54}$

Voigt $^{46}$

Haas ${ }^{60}$

Barre 55

Borstad 33

Bergqvist $^{32}$

Fricker ${ }^{36}$

Caen $^{35}$

EFS Group 39

Samama $60^{27}$

Samama $40^{27}$

Samama $20^{27}$

Briel $^{34}$

Verardi ${ }^{28}$

Chiapuzzo 51

Lassen $^{61}$

Planes $^{49}$

Adolf ${ }^{43}$

Kakkar $^{48}$

Heilmann $^{44}$

Dahan $^{40}$

Lassen $^{62}$

Dechanvanne ${ }^{56}$

Eriksson 57

Monreal $^{58}$

Pini $^{52}$

Baumgarten ${ }^{47}$

Hart| $\left.\right|^{37}$

Leizorovicz $^{4}$

Leyrraz 59

Levine ${ }^{50}$

the less, the data from these studies were not sufficien to allow any conclusions to be drawn about either the effect of low molecular weight heparin or the treatment schedule on major bleeding events as there were too few patients.

\section{CHRONOLOGICAL EVOLUTION OF RESULTS}

The chronological evolution of the odds ratios for deep vein thrombosis (both general and orthopaedic surgery) was investigated. After the disappointing results from the first study in 1984 using high doses and few patients the results seemed promising in 1985, and then the cumulative result became less significant in 1986 and 1987. From 1988 onwards the cumulative result showed an improvement, which seems to have remained stable, with the $95 \%$ confidence interval becoming smaller (fig 3), the odds ratio decreases drastically from 1984 to 1985 and then remain relatively stable, at between 0.5 to 0.9 . The number of patients included in the papers published in 1988 account for about half of the total number of patients and from this year onwards the odds ratio is apparently stable, at about $0 \cdot 8$. 
FIG 3-Graphical representation of results from meta-analysis (exact odds ratio) for risk of deep vein thrombosis in patients undergoing general and orthopaedic surgery. Odds ratio (-) is cumulative. From 1988 it is stable, around $0 \cdot 8$, and almost half of all the patients are included in this year. $95 \%$

Confidence intervals (horizontal lines) are reduced over time.

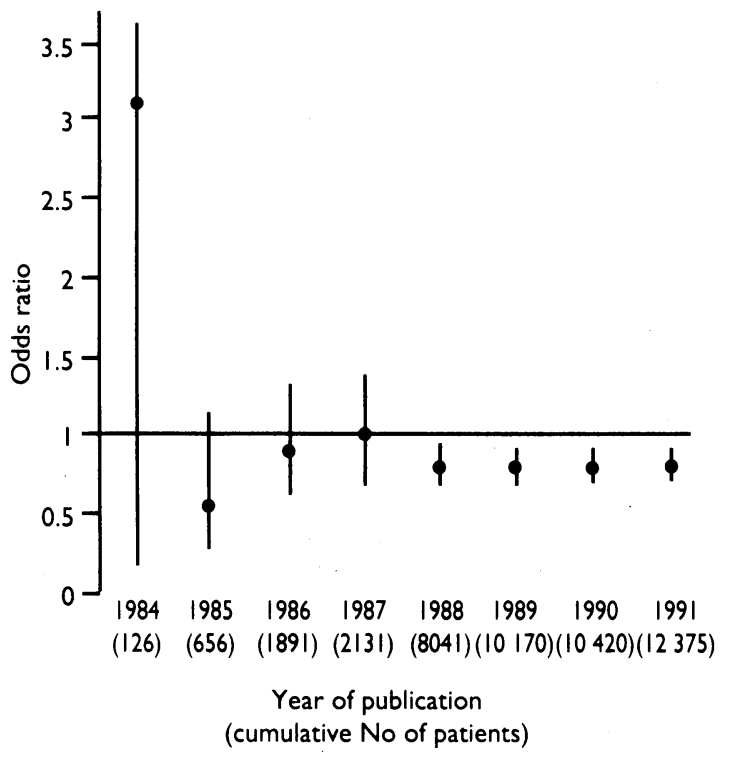

\section{Discussion}

The efficacy of low molecular weight heparins has been assessed in comparison with placebo and other prophylactic treatments, but in many cases the results have been ambiguous, often because of an inadequate sample size. If we assume that a clinically significant risk reduction would be a $30 \%$ difference in the incidence of deep vein thrombosis between the treatment and control groups for patients receiving general surgery, with a type I $(\alpha)$ error of $5 \%$ and a power $(1-\beta)$ of $95 \%$ in a two tailed test, we would need 9400 patients, given that the incidence of deep vein thrombosis is $5 \%$ with unfractionated heparin and $3.5 \%$ with low molecular weight heparin. The number of patients required if these incidences were $4 \%$ and $2 \cdot 8 \%$, respectively, would be 11900 . Thus no single study had a sample size sufficient to be able to detect a significant difference under this hypothesis.

Meta-analysis is a technique that allows the systematic, quantitative summary of data from individual studies, and it may supply the answer which individual trials cannot because of the increased statistical power afforded by the larger number of subjects. Even with the techniques used in meta-analysis, the power may remain too low so that no conclusions can be drawn, as was the case in previous meta-analyses. In our analysis there were insufficient data on the incidence of pulmonary embolism, so the analysis lacks power for this particular end point.

The efficacy of any meta-analysis can be influenced to a large extent by publication bias. This bias can arise when a study gives non-significant results, leading to reluctance by investigators and journal editors to submit and publish the results. ${ }^{70-73}$ Many studies are published only in the form of an abstract, which means that the methods are difficult to judge and the results are often only intermediate or not very detailed, or both. This point is important to remember when the results of a meta-analysis are considered. Some manufacturers seem to be reluctant to disclose data that have not already been divulged. In our search, thanks to one pharmaceutical firm, we located only one, small, unpublished study; perhaps others exist, but we were unable to locate them.

\section{COMPARISONS WITH PLACEBO, DEXTRAN, AND} UNFRACTIONATED HEPARIN

We identified only four studies comparing low molecular weight heparin with dextran in patients undergoing orthopaedic surgery, whereas placebo controlled studies were found for both general and orthopaedic surgery. Our results confirm previous findings that low molecular weight heparins are superior to placebo for the prophylactic treatment of deep vein thrombosis in patients who have had surgery. The results for pulmonary embolism and total mortality are not as convincing, mainly owing to insufficient numbers of patients.

Dextran has been widely used as a prophylactic treatment in general and orthopaedic surgery in Scandinavian countries. It was significantly superior to placebo in preventing deep vein thrombosis, pulmonary embolism, and death in clinical trials in more than 5000 patients. ${ }^{74}$ Dextran has also been compared with unfractionated heparin in a few studies, unfractionated heparin being more effective in patients undergoing general surgery and dextran in those undergoing orthopaedic surgery..$^{75}$ Our analysis sug- के gests that low molecular weight heparin is more $\vec{\circ}$ efficacious than dextran for the prophylactic treatment of deep vein thrombosis. We should be cautious, however, before extrapolating these results to pulmonary embolism and death because of the insufficient amount of data currently available.

We have shown that low molecular weight heparins, of compared with unfractionated heparins, reduce the 8 risk of deep vein thrombosis significantly in patients 0 undergoing both general and orthopaedic surgery. This end point was measured using various techniques, $\vec{\omega}$ but in many general surgery studies the technique used $ᄋ$ was the fibrinogen uptake test, which is not as accurate a diagnostic procedure as phlebography. The true positive rate for detecting thrombosis has been estimated as $99.6 \%$ and the false positive rate as about $9 \cdot 7 \%$ among patients undergoing general surgery. ${ }^{76}$ Although we used rates of phlebographically confirmed deep vein thrombosis in our analysis, when available, this close agreement between the methods suggests that the fibrinogen uptake test can give acceptably reliable estimates of the true postoperative rates of deep vein thrombosis. In the majority of the orthopaedic surgery studies phlebography was used for the confirmation of the diagnosis of deep vein thrombosis, which is important as fibrinogen uptake is less reliable in this indication.

\section{DOSAGE}

Although the adjusted dose regimen for unfractionated heparin may have a higher efficacy than a fixed dose regimen in orthopaedic surgery patients, ${ }^{778}$ this technique is not widely used, probably because of its 3 more onerous workload. This explains why we found only a few studies comparing adjusted dose heparin with low molecular weight heparin,,$^{53557}$ making it $\mathrm{O}$ impossible to compare these two treatments.

Controversial problems remain about the possibility of of a class effect and the lack of a standard for defining $\frac{D}{O}$ the concentrations and comparing the different preparations. The introduction of an international unit $N$ system - that is, antifactor Xa units - has reduced the $\mathrm{N}$ second problem, but this unit system is not always $\sigma$ quoted in publications, which can lead to difficulties 0 when comparing results from different studies. The official reference for the determination of the concen- $\stackrel{\mathscr{S}}{\rightarrow}$ tration in terms of anti-Xa units, was not always used, $T$ especially in the earlier trials, even when the concentration is quoted in these units. The low molecular weight heparins also differ in terms of molecular $\mathbb{\mathbb { Q }}$ weight distribution and the ratio of activities of anti-Xa and anti-IIa. This ratio is inversely proportional to the molecular weight and may be related to the antithrombotic activity of these products. Although the clinical relevance of these biochemical differences has $\frac{0}{7}$ not yet been established, it is perhaps incorrect to consider only the anti-factor Xa units when determining the prophylactic dose, but as the reports did not often 
give other values, we have used anti-Xa units when possible. The range of the observed $p$ values for heterogeneity $(0 \cdot 18-0 \cdot 84)$ does not suggest that the different low molecular weight heparins have quantitatively different effects. However, caution should be used when considering all these products as equal because only direct comparison, in a randomised controlled trial, will enable this hypothesis to be substantiated. Therefore, physicians wishing to use a low molecular weight heparin in their practice should take into consideration the results from the individual study in the choice of the appropriate drug and dose.

The prevention of deep vein thrombosis is important in patients at risk as deep vein thrombosis is a risk factor for pulmonary embolism. Although the incidence of pulmonary embolism is low, an efficient prophylatic treatment is needed because pulmonary embolism is sometimes disabling or fatal. The diagnosis of fatal and non-fatal pulmonary embolism is difficult and so we combined both in one end point. In an overview comparing unfractionated heparin with placebo the reduced incidence of deep vein thrombosis in heparin treated patients was similar to the observed reduction in the incidence of pulmonary embolism when the results were combined but not in individual trials. ${ }^{4}$ Therefore, the validity of deep vein thrombosis as a surrogate end point for pulmonary embolism has not yet been firmly established. We should consider the benefit to risk ratio because treatment with any type of heparin leads to increased risk of bleeding complications and, therefore, a patient's risk factors should be carefully examined before taking the decision to administer heparin.

The incidence of major bleeding might be reduced if the first injection is given a long time before or after the operation. In most of the studies identified for this analysis the initial injection was given two hours before surgery, although some had an initial injection at least 12 hours either before or after the operation. No conclusions about the effect of this on the incidence of bleeding could be drawn from the analysis performed with these available data because of the low power of the test, although a non-significant reduction was observed in trials with the first injection given 12 hours before surgery. A comparison of the incidence of bleeding in these different studies would be illegal, so it is not possible to say which is best, giving the first injection 12 or two hours before surgery or 12 hours after surgery; thus a large scale trial should be performed to answer this question.

A single daily dose of low molecular weight heparins certainly offers practical advantages to hospital staff and convenience for the patients. This is not sufficient because treatment is expensive, and therefore, we must also be sure that its efficacy is at least comparable, if not better, than that of existing treatments. Though an unequivocal answer has not been obtained in the individual studies, the results from our meta-analysis suggest that low molecular weight heparins are more efficacious than unfractionated heparins for the prophylactic treatment of deep vein thrombosis and pulmonary embolism

\section{CONCLUSIONS}

Meta-analysis is an undeniably powerful tool, but care should be taken when assessing the results from this type of analysis. In terms of the efficacy for prophylactic treatment of deep vein thrombosis, our results, though indicating the superiority of low molecular weight heparins over unfractionated heparins, cannot be used as a substitute for a large scale clinical trial. This polemic shows the value of metaanalysis when the end point of interest is rare or difficult to measure. A large scale randomised trial to assess the efficacy of this treatment in the prophylactic treatment of venous thrombosis in general and orthopaedic surgery with pulmonary embolism as the main end point needs to be performed. This would require the inclusion of several thousand patients, and two such trials with an expected sample size of 7500 patients are underway ${ }^{14}$ and may provide the data to confirm the prophylactic efficacy of heparin for this indication. In addition, a trial to investigate the benefits of low molecular weight heparin in preventing fatal pulmonary embolism and death from other causes is needed. This trial would be even larger, but is, none the less, feasible and well worth performing.

1 Dalen JE, Paraskos JA, Ockene IS, Alpert JS, Hirsh J. Venous thromboembolism: scope of the problem. Chest 1986;89(suppl):370-3S.

2 Hull RD, Raskob GE, Hirsh J. Prophylaxis of venous thromboembolism: an

overview. Chest $1986 ; 89$ (suppl):374-83S.
3 Kakkar VV. Prevention of venous thromboembolism. Clinical Haematolog 1981;10:543-82.

4 Collins R, Scrimgeour A, Yusuf S, Peto R. Reduction in fatal pulmonary embolism and venous thrombosis by perioperative administration of subcutaneous heparin. Overview of results of randomized trials in general, orthopedic and urologic surgery. $N$ Engl f Med 1988;318:1162-73.

5 Bergqvist $\mathrm{D}$. Dextran in the prophylaxis of deep-vein thrombosis. JAMA 1987;258:324-5.

6 Hirsh J. From unfractionated heparins to low molecular weight heparins. Act Chir Scand 1990;556(suppl):42-50.

7 Carter CJ, Kelton JG, Hirsh J, Cerskus A, Santos AV, Gent M. The relationship between the haemorrhagic and antithrombotic properties of low molecular weight heparin in rabbits. Blood 1982;59:1239-45.

8 Cade JF, Buchanan MR, Boneu B, Ockelford P, Carter CJ, Cekus AL, et al. A comparison of the antithrombotic and haemorrhagic effects of low molecular weight heparin fractions. Thromb Res 1984;35:613-25.

9 Buchanan MR, Boneu B, Ofosu FA, Hirsh J. The relative importance of thrombin inhibition and factor $\mathrm{Xa}$ inhibition to the antithrombotic effects $\mathrm{o}$ heparin. Blood 1985;65:198-201.

10 Esquivel CO, Bergqvist D, Biork CG, Nilsson B. Comparison between commercial heparin, low molecular weight heparin and pentosan polysulfate on haemostasis and platelets in vivo. Thromb Res 1982;28:389-99.

11 Lane DA, Denton J, Flynn AM, Thunberg L, Lindahl U. Anticoagulan activities of heparin oligosaccharides and their neutralization by platelet factor 4. Biochem f 1984;218:725-32.

12 Lockner D, Bratt G, Törnebohm E, Aberg W. Pharmacokinetics of intravenously and subcutaneously administered fragmin in healthy volunteers. Haemostasis 1986;16(suppl):8-10

13 Frydman AM, Bara L, Le Roux Y, Woler M, Chauliac F, Samama M. The antithrombotic activity and pharmacokinetics of enoxaparin, a low molecula weight heparin, in humans given single subcutaneous doses of 20 to $80 \mathrm{mg}$. J Clin Pharmacol 1988;28:609-18.

14 Boissel JP, Bossard N. Registry of multicenter clinical trials: eleventh report1989. Thromb Haemost 1991;66:368-83.

15 Boissel JP, Sacks HS, Leizorovicz A, Blanchard J, Panak E, Peyrieux JC Meta-analysis of clinical trials: summary of an international conference. Eur f Clin Pharmacol 1988; $34: 535-8$.

16 Thompson SG, Pocock SJ. Can meta-analyses be trusted? Lancet 1991;338: $1127-30$.

17 Fleiss J Statistical methods for rates and proportions. New York: Wiley, 1973.

18 Le Gagneux F, Steg A, Le Guillou M. Subcutaneous Enoxaparin (Lovenox ${ }^{n}$ ) versus placebo for preventing deep vein thrombosis (DVT) after transurethral prostatectomy (TUP). Thromb Haemost 1987:58:116.

19 Ockelford PA, Patterson J, Johns AS. A double-blind randomized placebo controlled trial of thromboprophylaxis in major elective general surger using once daily injections of a low molecular weight heparin fragment (Fragmin). Thromb Haemost 1989;62:1046-9.

20 Pezzuoli G, Neri Serneri GG, Settembrini P, Coggi G, Olivari N, Buzzetti G, et al. Prévention de l'embolie pulmonaire mortelle en chirurgie générale par le CY 216, héparine de bas poids moléculaire: essai multicentrique, randomisé, en double insu, contrôlé contre placebo (Etude STEP). Int Surg 1989;74:205-10.

21 Valle I, Sola G, Origone A. Controlled clinical study of the efficacy of a new low molecular weight heparin administered subcutaneously to prevent post operative deep venous thrombosis. Curr Med Res Opin 1988;11:80-6.

22 Turpie AGG, Levine MN, Hirsh J, Carter CJ, Jay RM, Powers J, et al. A randomized controlled trial of low molecular weight heparin (enoxaparin) to prevent deep vein thrombosis in patients undergoing elective hip surgery. $N$ Engl f Med 1986;315:925-9.

23 Leclerc J, Geerts W, Desjardins L, Jobin F, Delorme P, Bourgouin J. A randomized trial of enoxaparin for the prevention of deep vein thrombosis after major knee surgery. Thromb Haemost 1991;65:753

24 Jorgensen PS, Torholm C, Knudsen JB, Boeng L, Josefson L, Bjerregaard P, et al. Thromboprophylactic effect of low molecular weight heparin (Fragmin) in acute hip fracture surgery. A placebo controlled study. Thromb Haemos 1989;62:525.

25 Torholm C, Broeng L, Seest Jorgensen P, Bjerregaard P, Josephsen L, Korsholm Jorgensen $\mathrm{P}$, et al. Thromboprophylaxis by low-molecularweight heparin in elective hip surgery. I Bone foint Surg $[B r]$ 1991;73: 434-8.

26 Lassen ML, Borris LC, Christiansen HM, Olsen AD, Schott P, Eiskjaer S, al. Low molecular weight heparin in the prevention of thromboembolism in elective total hip replacement. Throm Haemost 1989;62:126.

27 Samama M, Bernard P, Bonnardot JP, Combe-Tamzali S, Lanson Y, Tissot E and GENOX. Low molecular weight heparin compared with unfractionated heparin in prevention of postoperative thrombosis. Br f Surg 1988;75: heparin

28 Veradi S, Casciani CU, Nicora E, Forzano F, Origone A, Valle I, et al. A multicentre study of LMW-heparin effectiveness in preventing postsurgical thrombosis. Int Angiol 1988;7:19-24.

29 Bergqvist D, Burmark US, Frisell J, Hallböök T, Lindblad B, Risberg B, et al. Low molecular weight heparin once daily compared with conventional lowdose heparin twice daily. A prospective double-blind multicentre trial on prevention of postoperative thrombosis. Brf S urg 1986;73:204-8. 
30 Koller M, Schoch U, Buchmann P, Largiadèr F, von Felten A, Frick PG. Low molecular weight heparin (KABI 2165) as thromboprophylaxis in elective visceral surgery. Thromb Haemost 1986;56:243-6.

31 Onarheim H, Lund T, Heimdal A, Arnesio B. A low molecular weight heparin (Kabi 2165 ) for prophylaxis of postoperative deep venous thrombosis. Acte Chir Scand 1986;152:593-6.

32 Bergqvist D, Mätzsch T, Burmark US, Frisell J, Guilbaud O, Hallböok T, et al. Low molecular weight heparin given the evening before surgery compared with conventional low-dose heparin in prevention of thrombosis. Br F Surg 1988;75:888-91.

33 Borstad E, Urdal K. Handeland G, Abildgaard U. Comparison of low molecular weight heparin vs. unfractionated heparin in gynecological surgery. Acta Obstet Gynecol Scand 1988;67:99-103.

34 Briel RC, Doller C, Hermann P. Thromboembolie-prophylaxe bei hysterektomien mit dem niedermolekularen heparin Fragmin. Geburtsch u Frauenheilk 1988;48: 160-4

35 Caen JP. A randomized double-blind study between a low molecular weight heparin Kabi 2165 and standard heparin in the prevention of deep vein thrombosis in general surgery. Thromb Haemost 1988;59:216-20.

36 Fricker JP, Vergnes Y, Schach R. Low dose heparin versus low molecula weight heparin $\left(\right.$ Kabi 2165, Fragmin ${ }^{k}$ ) in the prophylaxis of thromboembolic complications of abdominal oncological surgery. Eur $\mathcal{F}$ Clin Invest 1988;18: $561-7$.

37 Hartl P, Brücke P, Dienstl E, Vinazzer H. Prophylaxis of thromboembolism in general surgery: comparison between standard heparin and fragmin. Thromb Res 1990;57:577-84.

$38 \mathrm{Kakkar}$ VV, Murray. WJG. Efficacy and safety of low-molecular weight heparin (CY216) in preventing postoperative venous thrombo-embolism: a cooperative study. Br F Surg 1985;72:786-91.

39 European Fraxiparin Study (EFS) Group. Comparison of a low molecular weight heparin and unfractionated heparin for the prevention of deep vein thrombosis in patients undergoing abdominal surgery. Br F Surg 1988;75: 1058-63.

40 Dahan M, Levasseur P, Bogaty J, Boneu B, Samama M. Prevention of postoperative deep vein thrombosis (DVT) in malignant patients by Fraxiparin (a low molecular weight heparin). A cooperative trial. Thromb Haemost 1989;62:519.

41 Leizorovicz A, Picolet H, Peyrieux JC, Boissel JP, and the HBPM Research Group. Prevention of perioperative deep vein thrombosis in general surgery a multicentre double blind study comparing two doses of Logiparin and standard heparin. Br F Surg 1991;78:412-6.

42 Schmitz-Huebner U, Bünte H, Freise G, Reers B, Rüschemeyer C, Scherer R, et al. Clinical efficacy of low molecular weight heparin in postoperative thrombosis prophylaxis. Klin Wochenschr 1984;62:349-53.

43 Adolf J, Knee H, Roder JD, van de Flierdt E, Siewert JR. Thromboembolieprophylaxe mit niedermolekularem Heparin in der Abdominalchirurgie. Disch Med Wochenschr 1984;114:48-53.

44 Heilmann L, Kruck M, Schindler AE. Thromboseprophylaxe in der Gynäkologie: Doppleblind-Vergleich zwischen nierdermolekularem (LMWH) und unfraktioniertem (UFH) Heparin. Geburtsch u Frauenheilk 1989;49:803-7

45 Sasahara AA, Koppenhagen K, Häring R, Welzel D, Wolf H. Low molecular weight heparin plus dihydroergotamine for prophylaxis of postoperative deep vein thrombosis. BrF Surg 1986;73:697-700.

46 Voigt J, Hamelmann H, Hedderich J, Seifert J, Buchhammer T, Köhler A Wirksamkeit und unerwünschte wirkungen von neidermolekularem heparin-dihydroergotamin zur thromboembolieprophylaxe in der abdominalchirurgie. Zentralbl Chir 1986;111:1296-305.

47 Baumgartner A, Jacot N, Moser G, Krähenbühl B. Prevention of postoperative deep vein thrombosis by one daily injection of low molecular heparin and dihydroergotamine. Vasa 1989;18:152-6.

48 Kakkar VV, Stringer MD, Hedges AR, Parker CJ, Welzer D, Ward VP, et al. Fixed combinations of low-molecular weight or unfractionated heparin plus dihydroergotamine in the prevention of postoperative deep vein thrombosis. dihydroergotamine in the prove
Am $\mathcal{f}$ Surg 1989;157:413-8.

49 Planes A, Vochelle N, Mazas F, Mansat C, Zucman J, Landais A, et al. Prevention of postoperative venous thrombosis: a randomized trial comparing unfractionated heparin with low molecular weight heparin in patients undergoing total hip replacement. Thromb Haemost 1988;60:407-10.

50 Levine MN, Hirsh J, Gent M, Turpie AG, Leclerc J, Powers PJ, et al. Prevention of deep vein thrombosis after elective hip surgery. A randomized trial comparing low molecular weight heparin with standard unfractionated heparin. Ann Intern Med 1991;114:454-51.

51 Chiapuzzo E, Orengo GB, Ottria G, Chiapuzzo A, Palazzini E, Fusillo M. The use of low molecular weight heparins for post-surgical deep vein thrombosis prevention in orthopaedic patients. F Int Med Res 1988;16:359-66.

52 Pini M, Tagliaferri A, Manotti C, Lasagni F, Rinaldi E, Dettori AG. Low molecular weight heparin (Alfa LHWH) compared with unfractionated heparin in prevention of deep-vein thrombosis after hip fractures. Int Angiol 1989;8:134-9.

53 Haas S, Fritsche HM, Ritter H, Stemberger A, Wendt P, Zeisel HJ, et al. Thrombosis prophylaxis with low molecular weight heparin in total hip replacement. Thromb Haemost 1985;54:319.

54 Binsack T, Zellner M, Schimming I, Wirth C, Moser E, Riess H, et al. Thrombosis prophylaxis with $\mathrm{LMW}$ heparin and sodium heparin in patient with total hip replacement. Thromb Res 1986;suppl VI:83.

55 Barre J, Pfister G, Potron G, Droulle C, Baudrillard JC, Barbier et al. Efficacité et tolérance comparée du Kabi 2165 et de l'héparine standard dans la prévention des thromboses veineuses profondes au cours des prothèses totales de hanche. 7 Mal Vasc 1987;12:90-5.

56 Dechavanne $M$, Ville D, Berruyer $M$, Trepo F, Dalery F, Clermont N, et al. Randomized trial of low-molecular-weight heparin (Kabi 2165) versus adjusted-dose subcutaneous standard heparin in the prophylaxis of deepvein thrombosis after elective hip surgery. Haemostasis 1989;1:5-12.

57 Eriksson BI, Eriksson E, Wadenvik H, Tengborn L, Risberg B. Comparison of low molecular weight heparin and unfractionated heparin in prophylaxis of deep vein thrombosis and pulmonary embolism in total hip replacement. Thromb Haemost 1989;69:470.

58 Monreal M, Lafoz E, Navarro A, Granero X, Caja V, Caceres E, et al. A prospective double-blind trial of a low molecular weight heparin once daily compared with a conventional low-dose heparin three times daily to prevent pulmonary embolism and venous thrombosis in patients with hip fracture. pulmonary embolism and
J Trauma $1989 ; 29: 873-5$.

59 Leyvraz P, Bachmann F, Hoek J, Büller HR, Postel M, Samama M, et al.W Prevention of deep vein thrombosis after hip replacement: randomize comparison between unfractionated heparin and low molecular weight?. heparin. BMF 1991;303:543-8

60 Haas S, Stemberger A, Fritsche HM, Welzel D, Wolf H, Lechner F, et al. Prophylaxis of deep vein thrombosis in high risk patients undergoing total hip replacement with low molecular weight heparin plus dihydroergotamine. Arzneimittelforschung 1987;37:839-43.

61 Lassen MR, Borris LC, Christiansen HM, Moller-Larsen F, Knudsen VE, Boris $\mathrm{P}$, et al. Heparin/dihydroergotamine for venous thrombosis prophylaxis: comparison of low dose heparin and low molecular weight heparin in hip surgery. Brf S urg 1988;75:686-9.

62 Lassen MR, Borris LC, Christiansen HM, Moller-Larsen F, Knudsen VE, Boris $P$, et al. Prevention of thromboembolism in hip-fracture patients. Comparison of low-dose heparin and low-molecular-weight heparin combined with dihydroergotamine. Arch Orthop Trauma Surg 1989;108:10-3.

63 Danish Enoxaparin Study Group. Low-molecular-weight heparin (enoxaparin) vs Dextran 70. Arch Intern Med 1991;151:1612-24.

64 Eriksson BI, Zachrisson BE, Teger-Nilsson AC, Risberg B. Thrombosis prophylaxis with low molecular weight heparin in total hip replacement. Brf Surg 1988;75:1053-7.

65 Matzsch T, Bergqvist D, Fredin H, Hedner U. Safety and efficacy of a low molecular weight heparin (Logiparin) versus dextran as prophylaxis againstio thrombosis after total hip replacement. Acta Chir Scand 1988;543(suppl):C 80-4.

66 Matzsch T, Bergqvist D, Fredin H, Hedner U, Lindhagen A, Nistor L Comparison of the thromboprophylactic effect of a low molecular $-\vec{\omega}$ weight heparin versus dextran in total hip replacement. Thrombosis and Haemorrhagic Disorders 1991;3:25-9.

67 Hoffmann R, Largiadër F, Roethlin M. Perioperative Thromboembolieprophylaxe: niedrig dosiertes heparin oder low molecular weight heparin- $\vec{V}$ DHE, vor- und nachteile. Eine prospektive, randomisierte untersuchung. Helv Chir Acta 1987;54:521-5.

68 Breyer HG, Hahn F, Koppenhagen K, Bacher P, Werner B. Prevention of deep vein thrombosis in orthopedic surgery: Fragmin versus heparin-O DHE. Thromb Res 1987;suppl VII:23.

69 Collet JP, Boissel JP and the VALIDATA group. Sick population-treated population: the need for a better definition. Eur $\mathcal{f}$ Clin Pharmacole 1991;41:267-71

70 Simes RJ. Confronting publication bias: a cohort design for meta-analysis. Star. Med 1987;6:11-29.

71 Simes RJ. Publication bias: the case for an international registry of trials. $f$ Clin Oncol 1986;4:1529-41.

72 Dickersin K. Report from the Panel on the Case for Registers of Clinical Trials at the eighth annual meeting of the Society of Clinical Trials. Controlled Clin Trials 1988;9:76-81.

73 Easterbrook PJ, Berlin JA, Gopalan R, Matthews DR. Publication bias in clinical research. Lancet 1991;337:867-72.

74 Bergqvist D. Postoperative thromboembolism: frequency, etiology, prophylaxis. $\overrightarrow{\bar{O}}$ Berlin: Springer-Verlag, 1983:129-45.

75 Bergqvist D. Postoperative thromboembolism: frequency, etiology, prophylaxis. Berlin: Springer-Verlag, 1983:146-9.

76 Colditz GA, Tuden RL, Oster G. Rates of venous thrombosis after generaro surgery: combined results of randomized clinical trials. Lancet 1986;ii: $143-6$.

77 Leyvraz PF, Richard J, Bachmann F, Van Melle G, Treyvaud JM, Livio JJ, $e t$ al. Adjusted versus fixed-dose subcutaneous heparin in the prevention of deep-vein thrombosis after total hip replacement. $N$ Engl f Med 1983;309:O 954-8.

78 Taberner DA, Poller L, Thomson JM, Lemon G, Weighill FJ. Randomized study of adjusted versus fixed low dose heparin prophylaxis of deep vein thrombosis in hip surgery. Br $\mathcal{F}$ Surg 1989;76:933-5.

(Accepted 23 fuly 1992)

\section{Correction}

The role of non-steroidal anti-inflammatory drugs in acute liver injury

An authors' error occurred in this paper by García Rodríguez et ab (10 October, p 866). In table II there should be no reference to sulindac in line seven, which should read:

Indomethacin $\quad 1 \quad 100 \quad 1982 \quad$ M 76 Yes Hepatocellular $\$$ This does not affect the information in other tables or in the text 